\title{
Giant Bubble Pinch-Off
}

\author{
Raymond Bergmann, ${ }^{1}$ Devaraj van der Meer, ${ }^{1}$ Mark Stijnman, ${ }^{1}$ Marijn Sandtke, ${ }^{1}$ Andrea Prosperetti, ${ }^{1,2}$ and Detlef Lohse ${ }^{1}$ \\ ${ }^{1}$ Physics of Fluids Group and J. M. Burgers Centre for Fluid Dynamics, Department of Science and Technology, University of Twente, \\ P.O. Box 217, 7500 AE Enschede, The Netherlands \\ ${ }^{2}$ Department of Mechanical Engineering, The Johns-Hopkins University, Baltimore, Maryland 21218, USA
}

(Received 23 December 2005; published 21 April 2006)

\begin{abstract}
Self-similarity has been the paradigmatic picture for the pinch-off of a drop. Here we will show through high-speed imaging and boundary integral simulations that the inverse problem, the pinch-off of an air bubble in water, is not self-similar in a strict sense: A disk is quickly pulled through a water surface, leading to a giant, cylindrical void which after collapse creates an upward and a downward jet. Only in the limiting case of large Froude numbers does the purely inertial scaling $h(-\log h)^{1 / 4} \propto \tau^{1 / 2}$ for the neck radius $h$ [J. M. Gordillo et al., Phys. Rev. Lett. 95, 194501 (2005)] become visible. For any finite Froude number the collapse is slower, and a second length scale, the curvature of the void, comes into play. Both length scales are found to exhibit power-law scaling in time, but with different exponents depending on the Froude number, signaling the nonuniversality of the bubble pinch-off.
\end{abstract}

PACS numbers: 47.55.df, 47.20.Ma, 47.55.db

The pinch-off of a liquid drop is a prime example of a hydrodynamic singularity and has been studied extensively in recent years [1-4]. It has become paradigmatic for selfsimilar behavior in fluid dynamics: After appropriate rescaling, the shapes of the pinching neck at different times can be superimposed onto a single shape [5-8]. With the exception of some pioneering work $[9,10]$, the inverse problem of the collapse of a gas-filled neck surrounded by a liquid has not attracted much attention until very recently, with the analysis of the pinch-off of a bubble rising from a needle and the breakup of a gas bubble in a straining flow [11-14]. The time evolution of these collapsing gas-filled necks is found to follow a power law. If the dynamics near the singularity are solely governed by liquid inertia, then the radius of the neck $h$ expressed in the time $\tau$ remaining until collapse scales as $h \propto \tau^{1 / 2}[9,10,12]$, or, with a $\log$ arithmic correction, as $h(-\log h)^{1 / 4} \propto \tau^{1 / 2}$ [11]. Deviations from this exponent of $1 / 2$ are reported to occur only due to the inclusion of other effects. The collapse may be slowed down by viscosity ( $h \propto \tau[12,13])$ or surface tension $\left(h \propto \tau^{2 / 3}[14]\right)$, or accelerated by the inertia of the gas flowing inside the neck, leading to $h \propto \tau^{1 / 3}$ [11].

In this Letter we focus on another example of this "inverse pinch-off", namely, the violent collapse of the void created at a fluid surface by the impact of an object. Here we find exponents which deviate substantially from $1 / 2$, even though the dynamics are shown to be purely governed by liquid inertia, without significant contributions from the effects mentioned above. The self-similar behavior $h(-\log h)^{1 / 4} \propto \tau^{1 / 2}$ appears to hold only in the asymptotic regime of very high impact velocities.

In our experiment, a linear motor is used to drag metal disks with radii $h_{\text {disk }}$ between 10 and $40 \mathrm{~mm}$ through an airwater interface with a well-controlled, constant velocity $V$ between 0.5 and $3 \mathrm{~m} / \mathrm{s}$ [see Fig. 1(a)]. A rod running through a seal in the bottom of a large water tank connects the disk with the linear motor. This arrangement generates giant voids in a very controlled fashion. The advantage of this setup is that the velocity is a control parameter and not the response of the object to the fluid forces upon impact. Second, due to the large scale of the experiment, viscosity and surface tension play a negligible role [15]. Therefore the only important dimensionless parameter is the Froude number $\mathrm{Fr}=V^{2} /\left(h_{\text {disk }} g\right)$, the ratio of kinetic to gravitational energy, which ranges from 0.6 to 90 . The large scale of the experiment is also advantageous for the observation of details during the impact and collapse process, which is imaged with digital high-speed cameras with frame rates up to 100000 frames per second.

A typical series of events is seen in Figs. 1(a)-1(d). The impact of the disk creates an axisymmetric void which first expands until the hydrostatic pressure drives the walls inward. The inward moving walls collide and cause a pinch-off at some depth below the undisturbed free surface. The energy focusing of this violent collapse creates a strong pressure spike on the axis of symmetry which releases itself in a downward and an upward jet $[16,17]$. The latter reaches heights exceeding $1.5 \mathrm{~m}$ for the higher impact speeds in this experiment. It is this dominating role of inertia that makes our system different from other pinchoff processes in the literature. At higher recording speeds the pinch-off can be investigated in more detail as in Figs. 1(e) $-1(\mathrm{~h})$. There is a clear loss of both azimuthal and axial symmetry in Figs. 1(f) and 1(g) which can be attributed to a combination of the same convergence effect that causes an instability in a collapsing bubble [18-20], and a Kelvin-Helmholtz instability due to the rapid air flow in the neck. The latter increases with increasing Froude number and limits the range of our experiments. Another factor which limits the Froude number range is the socalled surface seal, in which the void closes at the water surface as the crownlike splash is entrained by the air flowing into the expanding void $[21,22]$. This process, which 

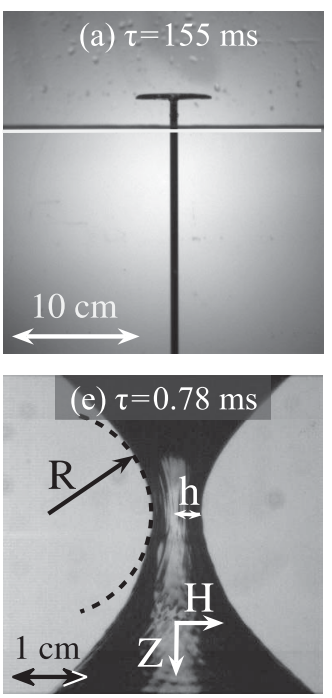
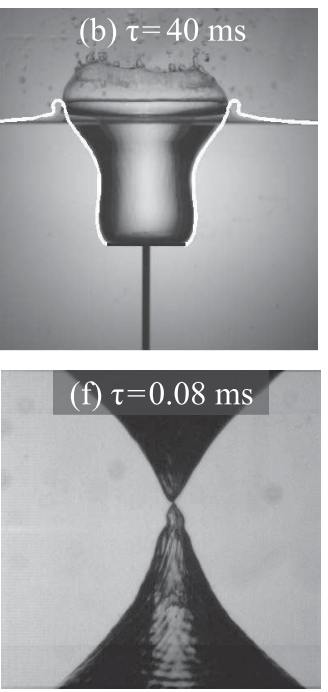
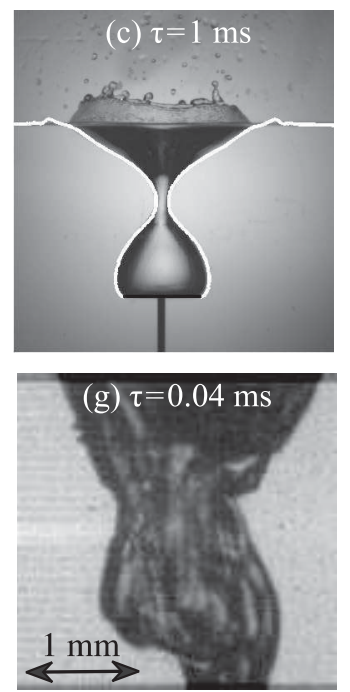
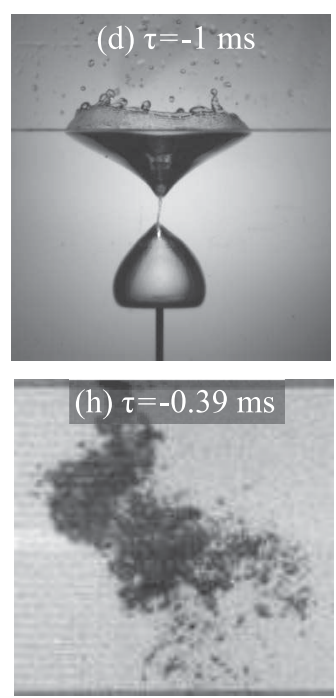

FIG. 1. Snapshots of the formation and collapse of a surface void for the plunger experiment: A linear motor pulls down a disk of radius $h_{\text {disk }}=30 \mathrm{~mm}$ through the water surface at a constant velocity $V=1.0 \mathrm{~m} / \mathrm{s}(\mathrm{Fr}=3.4)$. (a-d) Collapse of the void imaged at a 1000 frames per second (fps). The white lines (overlay) are the void profiles obtained from boundary integral simulations with the same initial condition, and without the use of any free parameter. (e-f) Details of the collapse imaged at $12800 \mathrm{fps}$. (g-h) Details of the collapse imaged at $48000 \mathrm{fps}$. Note that the field of view decreases with increasing frame rate. In the very last stages of the collapse $(\mathrm{f}-\mathrm{g})$ there is a Kevin-Helmholtz instability that complicates the determination of the time of collapse. Immediately after the collapse air is entrapped, both in the form of a large bubble above disk (d) and as a cloud of microbubbles at the collapse point (h).

occurs at large Froude numbers, changes the pinch-off considerably since in this case the gas pressure inside the void differs appreciably from that of the ambient air.

In view of these experimental limitations, we performed numerical simulations using a boundary integral method based on potential theory without ambient gas [23]. There is an excellent agreement between the numerical calculations and the experiments, as seen in Figs. 1(a)-1(c). Here, the numerical void profiles (the solid white lines) coincide very well with the experimental profiles in the pinch-off region without the use of any adjustable parameter, either in space or in time.

To further quantify the pinch-off process, we now turn to the time evolution of the neck radius $h(\tau)$, measured at the depth at which the void eventually closes. Since both length and time scales become very small close to collapse, it is not feasible to experimentally observe the collapse with only one high-speed camera recording [24]. Because of the reproducibility of the experiment, we could overcome this difficulty by matching several data sets imaged at different frame rates, increasingly magnifying the region around the pinch-off. Figure 2(a) contains a doubly logarithmic plot of $h(\tau)$ for both the high-speed imaged experiments and the numerical calculations, again showing excellent agreement for different Froude numbers. There is a clear power-law behavior. Compensating the data with $\tau^{1 / 2}$ [Fig. 2(b)] and subsequently plotting the corresponding power-law exponent $\alpha_{h}$ as a function of Fr [Fig. 2(d)] reveal large deviations from the suggested $1 / 2$ power law. Can these be explained by a logarithmic correction as proposed in [11]?
Let us first establish the origin of this logarithmic correction in our system. Near the neck, the flow induced by the collapsing void looks very much like that of a collapsing cylinder, while it must look like that of a sink, plus its image in the free surface (i.e., a dipole) in the far region. In the language of singular perturbations, the former would be the inner region and the latter the outer region; a complete description would require the matching of these two regions. If we disregard the outer region, we can use a twodimensional version of the Rayleigh equation, which describes the collapse of an infinite cylindrical cavity under uniform pressure $[10,17,25]$

$$
\left[\frac{d(h \dot{h})}{d \tau}\right] \log \frac{h}{h_{\infty}}+\frac{1}{2} \dot{h}^{2}=g Z \text {. }
$$

The pressure difference driving the collapse has been equated to $\rho g Z$, where $Z$ denotes the depth below the fluid surface, which implies that the system is composed of noninteracting horizontal layers of fluid, with a negligible vertical velocity component [26]. Although $h_{\infty}$ (the radius at which both pressure and velocity are quiescent) must in principle be determined by the matching process alluded to before, it is expected to be of the order of a typical length scale of the process, such as the cavity depth. Thus, strictly speaking, $h_{\infty}$ is a function of time and of the Froude number. However, near pinch-off, the time scale for the neck motion is much faster than that for the evolution of the other parts of the cavity so that $h_{\infty}$ may be considered only a function of Fr. After an initial expansion of the void, the collapse starts from rest at a maximal radius $h_{\max }$ (of the order of $h_{\text {disk }}$ ). Using this as an initial condition, and 

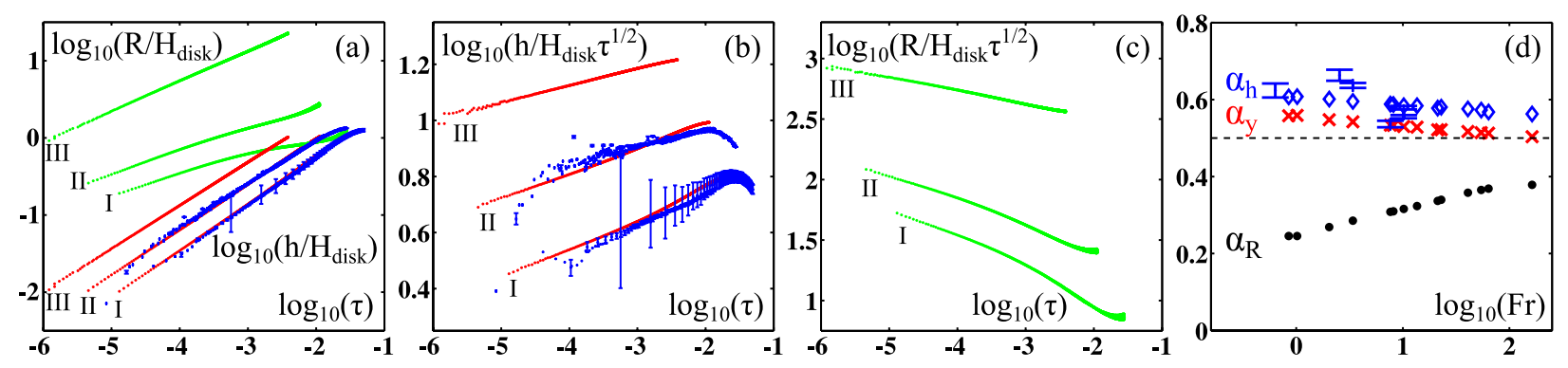

FIG. 2 (color online). (a) The radius of the void at the depth of closure $h$ (lower three curves) and the radius of curvature of the void profile $R$ (upper three curves) as a function of the time $\tau$ remaining until collapse in a doubly logarithmic plot, for Fr $=3.4$ (I), $\mathrm{Fr}=10.2$ (II), and $\mathrm{Fr}=163$ (III). Both $h$ and $R$ are well described by power laws for up to 4 orders of magnitude in $\tau$. Experiment (blue dots) and numerical simulations (red dots) agree very well for Fr $=3.4$ and 10.2. For Fr $=163$ only numerical data are presented, because here experiments are hindered by the surface seal (see text). The error bars, indicating the error in the experimental data, are usually small, but occasionally become large for frames very close to the collapse time. (b) Doubly logarithmic plot of the void radius $h$ compensated with $\tau^{1 / 2}$ to better show the deviations from the expected power-law behavior. (c) Doubly logarithmic plot of the radius of curvature $R$ compensated with $\tau^{1 / 2}$ for the numerical simulations of (a-b). (d) Power law exponents $\alpha_{h}$ for the radius of the void at closure depth $h(\tau), \alpha_{y}$ for the radius of the void including the logarithmic correction $y(\tau)=$ $h(-\log h)^{1 / 4}$, and $\alpha_{R}$ for the radius of curvature $R$, all as a function of the Froude number.

treating $h_{\infty}$ as a constant, the energy integral of Eq. (1) can be readily found:

$$
\left(\frac{d \tilde{h}}{d \tilde{\tau}}\right)^{2}=\frac{1}{\log (\tilde{h} / \tilde{h} \infty)}\left[1-(1 / \tilde{h})^{2}\right]
$$

where we have introduced the nondimensional variables $\tilde{h} \equiv h / h_{\max }, \tilde{h}_{\infty} \equiv h_{\infty} / h_{\max }$, and $\tilde{\tau} \equiv \tau \sqrt{g Z / h_{\max }^{2}}$. Close to pinch-off, $\tilde{h}^{2} \ll 1$, such that $\tilde{h}^{-2}-1 \approx \tilde{h}^{-2}$. With this approximation, we can integrate Eq. (2) once more to arrive at

$$
2 \tilde{\tau}=\tilde{h}^{2} \sqrt{\log \left(\tilde{h}_{\infty} / \tilde{h}\right)}+\sqrt{\frac{\pi}{2}} \tilde{h}_{\infty}^{2} \operatorname{erfc}\left(\sqrt{2 \log \left(\tilde{h}_{\infty} / \tilde{h}\right)}\right) .
$$

For small $\tilde{h}$ the term with the complementary error function is always small compared to the first one and their ratio vanishes for $\tilde{h} \downarrow 0$. Neglecting this term we find two asymptotic regimes

$$
\tilde{h}\left[\log \left(\tilde{h}_{\infty}\right)\right]^{1 / 4}=\sqrt{2} \tilde{\tau}^{1 / 2} \quad \text { for } \tilde{h} \gg 1 / \tilde{h}_{\infty},
$$

and

$$
\tilde{h}[-\log (\tilde{h})]^{1 / 4}=\sqrt{2} \tilde{\tau}^{1 / 2} \text { for } \tilde{h} \ll 1 / \tilde{h}_{\infty}
$$

From Eqs. (4) and (5) we conclude that the scaling depends crucially on the value of $h_{\infty}$ : Initially, for the intermediate regime $h \gg h_{\max }^{2} / h_{\infty}$, we expect to find a strict power law $h \propto \tau^{1 / 2}$, since $\log \left(\tilde{h}_{\infty}\right)$ is constant. For times closer to the pinch-off, when $h \ll h_{\max }^{2} / h_{\infty}$, logarithmic corrections play a role, and the power law should be modified into Eq. (5).

As $h_{\infty} \approx h_{\max }$ in our experiments, the latter inequality can be read as $h \ll h_{\max }$, which is satisfied in most of the region where $h(\tau)$ asymptotically behaves as a power law [cf. Figs. 2(a) and 2(b)]. We conclude that in our system the logarithmic correction cannot be neglected. If we plot the quantity $y=h(-\log h)^{1 / 4}$ vs time, we again observe a power law $y \propto \tau^{\alpha_{y}}$, but with a slightly different exponent $\alpha_{y}$ than the one found for $h$. In Fig. 2(d) we compare $\alpha_{h}$ and $\alpha_{y}$ as functions of the Froude number. As discussed before, $\alpha_{h}$ is substantially larger than $1 / 2$, but even if the logarithmic term is included we continue to find a slower collapse for low Froude numbers. Although the logarithmic correction does bring the result closer to the suggested value $1 / 2$, it cannot account for all of the observed deviations.

Clearly, the observed anomalous power law behavior of the neck radius must reflect itself in the time evolution of the free-surface profiles of the collapsing void. If the process were self-similar, the free-surface profiles at different times $\tau$ would superpose when scaled by any characteristic length, e.g., the neck radius $h$. Actually, it is found that the depth of minimum radius increases somewhat as the collapse progresses and it is therefore necessary to translate the profiles in the vertical direction so as to match the position of the minimum radius point before attempting this operation. Even if this is done, however, the results fail to collapse onto a single shape when using a single length scale. The rescaled profiles become more and more elongated as the pinch-off is approached which proves that the collapsing void is not self-similar in a strict sense.

The free-surface shapes near the minimum point should thus not only be characterized by $h(\tau)$, but also by a second length scale, the radius of curvature $R(\tau)$ in the vertical plane [see Fig. 1(e)]. The spatial resolution of the highspeed camera images limits the accuracy with which $R$ can be extracted from the experimental observations, but this quantity is easily determined from the numerical calculations [see Figs. 2(a) and 2(c)]. When the radial dimensions [H, cf. Fig. 1(e)] are scaled by $h$ and vertical ones [Z] by $\sqrt{h R}$, the profiles do collapse onto a single curve. This, however, may only signal that their shape is very close to parabolic [27]. The time evolution of this radius of curvature is also found to follow a power law, $R=R_{0} \tau^{\alpha_{R}}$, the 
exponent $\alpha_{R}$ of which increases with the Froude number as can be seen in Fig. 2(d) [28].

The essence of the time evolution of the void profile and the departure from self-similarity in the strict sense is captured in the aspect ratio $h / R$ of the collapsing void, $h(\tau) / R(\tau)=\left(h_{0} / R_{0}\right) \tau^{\left(\alpha_{h}-\alpha_{R}\right)}$, in which the prefactor $h_{0} /$ $R_{0}$ and the exponent $\left(\alpha_{h}-\alpha_{R}\right)$ both are found to depend on the Froude number. It is seen in Fig. 2(d) that $\alpha_{h}-$ $\alpha_{R}>0$ for any finite Froude number, causing the ratio $h(\tau) / R(\tau)$ to vanish in the limit $\tau \rightarrow 0$. This means that in this limit $R(\tau)$ becomes large with respect to the neck radius, elongating the profiles more and more towards the cylindrical shape close to the pinch-off, thereby justifying the assumptions made in the derivation of Eq. (1) in the limit $\tau \rightarrow 0$. A numerical fit gives $\left(\alpha_{h}-\alpha_{R}\right) \propto \mathrm{Fr}^{-0.14}$, which indicates that $h$ and $R$ have the same time dependence as $\mathrm{Fr} \rightarrow \infty$ and, therefore, that self-similarity is recovered in this limit. A second numerical fit shows that $h_{0} / R_{0} \propto \mathrm{Fr}^{-0.60}$, which tends to zero as $\mathrm{Fr} \rightarrow \infty$. This feature expresses the experimental observation that the initial elongation of the neck is larger for large Froude numbers, which effectively increases the time interval for which the assumption of pure radial flow is valid [cf. Eq. (1)].

In conclusion, our experiments on the collapse of a giant surface void are in excellent agreement with boundary integral calculations without the use of any adjustable parameter. Even when we exclude the effects of air, viscosity, and surface tension, the collapse is found to be not self-similar in a strict sense, but governed by power laws with nonuniversal, Froude-dependent exponents. Very near to pinch-off the void profile approaches the cylindrical shape, a regime that is approached earlier as the Froude number is larger. Self-similarity is recovered only in the limit of infinite Froude number, where the influence of gravity becomes negligible and the collapse is truly inertially driven.

We thank J. M. Gordillo, S. R. Nagel, and W. W. Zhang for many discussions and for sharing their results prior to publication. This work is part of the research program of the Stichting FOM, which is financially supported by NWO.

[1] A. L. Bertozzi, M.P. Brenner, T.F. Dupont, and L.P. Kadanoff, in Trends and Perspectives in Applied Mathematics, edited by L. Sirovich (Springer, New York, 1993), Vol. 100, p. 155.

[2] H. A. Stone, Annu. Rev. Fluid Mech. 26, 65 (1994).

[3] M. P. Brenner, X. D. Shi, and S. R. Nagel, Phys. Rev. Lett. 73, 3391 (1994)

[4] P. Doshi, I. Cohen, W. W. Zhang, M. Siegel, P. Howell, O. A. Basaran, and S. R. Nagel, Science 302, 1185 (2003).

[5] J. Eggers, Rev. Mod. Phys. 69, 865 (1997).

[6] R. F. Day, E. J. Hinch, and J. R. Lister, Phys. Rev. Lett. 80, 704 (1998).

[7] W. W. Zhang and J. R. Lister, Phys. Rev. Lett. 83, 1151 (1999).
[8] I. Cohen, M. P. Brenner, J. Eggers, and S. R. Nagel, Phys. Rev. Lett. 83, 1147 (1999).

[9] M.S. Longuet-Higgins, B. R. Kerman, and K. Lunde, J. Fluid Mech. 230, 365 (1991).

[10] H. N. Oguz and A. Prosperetti, J. Fluid Mech. 257, 111 (1993).

[11] J. M. Gordillo, A. Sevilla, J. Rodriguez-Rodriguez, and C. Martinez-Bazan, Phys. Rev. Lett. 95, 194501 (2005).

[12] J. C. Burton, R. Waldrep, and P. Taborek, Phys. Rev. Lett. 94, 184502 (2005).

[13] R. Suryo, P. Doshi, and O. A. Basaran, Phys. Fluids 16, 4177 (2004).

[14] D. Leppinen and J. R. Lister, Phys. Fluids 15, 568 (2003).

[15] Viscosity and surface tension effects are quantified by the magnitude of the Reynolds (Re) and Weber (We) numbers, which are considerable $\left(>10^{2}\right)$ during the pinch-off process. This holds when they are defined globally, i.e., with respect to the impact velocity and the disk radius $(\mathrm{Re}=$ $h_{\text {disk }} V / \nu$ and $\left.\mathrm{We}=h_{\text {disk }} V^{2} \rho / \sigma\right)$, but also when they are defined locally using the neck radius and velocity at a specific time $\left(\operatorname{Re}=h \dot{h} / \nu\right.$ and $\left.\mathrm{We}=h \dot{h}^{2} \rho / \sigma\right)$.

[16] B. W. Zeff, B. Kleber, J. Fineberg, and D. P. Lathrop, Nature (London) 403, 401 (2000).

[17] D. Lohse et al., Phys. Rev. Lett. 93, 198003 (2004).

[18] M.S. Plesset and A. Prosperetti, Annu. Rev. Fluid Mech. 9, 145 (1977).

[19] Y. Hao and A. Prosperetti, Phys. Fluids 11, 1309 (1999).

[20] S. Hilgenfeldt, D. Lohse, and M. P. Brenner, Phys. Fluids 8, 2808 (1996).

[21] G. Birkhoff and E.H. Zarantonello, Jets, Wakes, and Cavities. (Academic, New York, 1957).

[22] S. Gaudet, Phys. Fluids 10, 2489 (1998).

[23] The ambient air will be included in a forthcoming publication, also using the boundary integral method.

[24] We image the pinch-off process over 4 orders of magnitude in time and two in space. As the field of view of the camera corresponds to $10^{3}$ pixels, this would leave only 10 pixels for the last stage of the collapse. The whole sequence should then be imaged at a frame rate corresponding to the smallest time scale $(10 \mu$ s), i.e., $100 \mathrm{kHz}$ requiring at least $10 \mathrm{~GB}$ of fast storage capacity, greatly exceeding the physical capabilities of our cameras.

[25] A. Prosperetti, Phys. Fluids 16, 1852 (2004).

[26] A similar equation is used in [11], without the parameter $h_{\infty}$ and also without the hydrostatic driving pressure $g Z$.

[27] At the minimum $1 / R(\tau)=d^{2} H /\left.d Z^{2}\right|_{Z=Z_{\min }}$ and the shape of the interface can be taken to be locally parabolic, which implies $H=(\delta Z)^{2} / R(\tau)+h(\tau)$ with $\delta Z=Z-Z_{\min }$. The scaling of the radial direction $H$ with $h(\tau)$ then leads to the scaling $\sqrt{h(\tau) R(\tau)}$ for the axial direction $Z$. The aspect ratio of the void is then given by $H / Z=$ $[h(\tau) / R(\tau)]^{1 / 2}$.

[28] The fact that both $h$ and $R$ are described by power laws suggests that we may be dealing with self-similarity of the second kind, in which the radial and axial coordinates are rescaled by different power laws of time [29]. At present there is however insufficient experimental and theoretical ground to substantiate such a claim.

[29] G. I. Barenblatt, Scaling, Self-Similarity, and Intermediate Asymptotics (Cambridge University Press, Cambridge, England, 1996). 